\title{
Generation of Distinct Types of Periglomerular Olfactory Bulb Interneurons during Development and in Adult Mice: Implication for Intrinsic Properties of the Subventricular Zone Progenitor Population
}

\author{
Silvia De Marchis, ${ }^{1}$ Serena Bovetti, ${ }^{1,2 *}$ Barbara Carletti, ${ }^{3 *}$ Yi-Chun Hsieh, ${ }^{2}$ Donatella Garzotto, ${ }^{1}$ Paolo Peretto, ${ }^{1}$ \\ Aldo Fasolo, ${ }^{1}$ Adam C. Puche, ${ }^{2}$ and Ferdinando Rossi ${ }^{3}$ \\ ${ }^{1}$ Department of Animal and Human Biology, University of Turin, I-10123 Turin, Italy, ${ }^{2}$ Department of Anatomy and Neurobiology, University of Maryland \\ School of Medicine, Baltimore, Maryland 21201, and '3Department of Neuroscience, University of Turin, I-10125 Turin, Italy
}

\begin{abstract}
The subventricular zone (SVZ) of the lateral ventricle develops from residual progenitors of the embryonic lateral ganglionic eminence (LGE) and maintains neurogenic activity throughout life. Precursors from LGE/SVZ migrate to the olfactory bulb (OB) where they differentiate into local interneurons, principally in the granule layer and glomerular layer (GL). By in situ dye labeling, we show that neonatal and adult SVZ progenitors differentially contribute to neurochemically distinct types of periglomerular interneurons in the GL. Namely, calbindin-positive periglomerular cells are preferentially generated during early life, whereas calretinin- and tyrosine hydroxylase-expressing neurons are mainly produced at later ages. Furthermore, homochronic/heterochronic transplantation demonstrates that progenitor cells isolated from the LGE or SVZ at different stages (embryonic day 15 and postnatal days 2 and 30) engraft into the SVZ of neonatal or adult mice, migrate to the $\mathrm{OB}$, and differentiate into local interneurons, including granule and periglomerular cells as well as other types of interneurons. The total number of integrated cells and the relative proportion of granule or periglomerular neurons change, according to the donor age, whereas they are weakly influenced by the recipient age. Analysis of the neurochemical phenotypes acquired by transplanted cells in the GL shows that donor cells of different ages also differentiate according to their origin, regardless of the host age. This suggests that progenitor cells at different ontogenetic stages are intrinsically directed toward specific lineages. Neurogenic processes occurring during development and in adult $\mathrm{OB}$ are not equivalent and produce different types of periglomerular interneurons as a consequence of intrinsic properties of the SVZ progenitors.
\end{abstract}

Key words: olfactory bulb; subventricular zone; transplantation; periglomerular cell; specification; neurogenesis

\section{Introduction}

The olfactory bulb $(\mathrm{OB})$ is one of the two regions in the mammalian brain in which neurogenesis persists throughout life (Alvarez-Buylla and Lim, 2004). The germinal zone responsible for this activity is the subventricular zone (SVZ), which lies adjacent to the lateral wall of the telencephalic ventricle. The SVZ develops from residual progenitors of the lateral ganglionic eminence (LGE) (Wichterle et al., 2001). The postnatal SVZ shows similar but restricted lineage potentiality compared with the LGE, which is the origin of both OB interneurons and striatal

Received July 6, 2006; revised Nov. 28, 2006; accepted Dec. 4, 2006.

This work was supported by Istituto Superiore di Sanità National Program on Stem Cells (CS107.1) (F.R.), Compagnia di San Paolo (NEUROTRANSPLANT), Regione Piemonte Ricerca Scientifica Applicata (Comitato Interministeriale per la Programmazione Economica) (Project A14) and Progetti di Rilevante Interesse Nazionale 2005 (Project 2005055095) (F.R., A.F., P.P.), and National Institute on Deafness and Other Communication Disorders DC05739 (A.C.P.). B.C. is supported by a fellowship funded by Michele and Marina Petochi.

*S.B. and B.C. contributed equally to this work.

Correspondence should be addressed to Dr. Silvia De Marchis, Department of Animal and Human Biology, University of Turin, Via Accademia Albertina 13, I-10123 Turin, Italy. E-mail: silvia.demarchis@unito.it.

DOI:10.1523/JNEUROSCI.2870-06.2007

Copyright $\odot 2007$ Society for Neuroscience $\quad$ 0270-6474/07/270657-08\$15.00/0 projection neurons (Wichterle et al., 2001; Stenman et al., 2003). SVZ-derived precursors migrate tangentially along the rostral migratory stream (RMS) to the OB, where they differentiate into local interneurons, within the granule (GCL) and glomerular (GL) layers (Luskin, 1993; Lois and Alvarez-Buylla, 1994). Interneurons of the GL and GCL modulate the activity of major output neurons in the $\mathrm{OB}$ and play essential roles in olfactory information processing (for review, see Shipley et al., 2004). Functional integration of newly generated neurons into the adult OB circuitry has been recently demonstrated (Belluzzi et al., 2003; Carleton et al., 2003). Nonetheless, the function of olfactory interneuron turnover in the adult brain remains elusive.

OB interneurons consist of a wide variety of phenotypes that differ in their spatial distribution, neurochemistry, structural features, and synaptic properties (Kosaka et al., 1998; Shipley et al., 2004; Kosaka and Kosaka, 2005; Parrish-Aungst et al., 2007; Pressler and Strowbridge, 2006). Major categories of local OB interneurons are granule (GC) and periglomerular (PG) cells. PG cells, in particular, can be further subdivided in several subclasses based on specific expression of neurochemical markers, including neurotransmitters and calcium-binding proteins (Kosaka 
and Kosaka, 2005; Parrish-Aungst et al., 2007). A certain degree of molecular heterogeneity already exists in migrating SVZ neuroblasts (Jankovski and Sotelo, 1996; Baker et al., 2001; De Marchis et al., 2004), and recent reports show that both intrinsic (Hack et al., 2005; Kohwi et al., 2005; Waclaw et al., 2006) and extrinsic mechanisms (Hack et al., 2005) are involved in fate determination of $\mathrm{OB}$ interneurons. Recent findings suggest that adult born granule cells have distinct properties compared with those generated during early postnatal life (Lemasson et al., 2005; Magavi et al., 2005). However, it is unclear whether all of the different categories of $\mathrm{OB}$ interneurons are continuously produced throughout life or whether distinct subtypes are generated within specific developmental windows. To address this question, we labeled in situ or transplanted progenitor cells from embryonic, postnatal, and adult SVZ, and assessed their ability to differentiate into different classes of $\mathrm{OB}$ interneurons, with special attention on PG phenotypes. Our results indicate that LGE/ SVZ progenitors preferentially generate certain subtypes of $\mathrm{PG}$ interneurons according to their age or donor origin. Together, these observations suggest that the neurochemically heterogeneous subpopulations of $\mathrm{OB}$ interneurons are produced in a precise time sequence by modulating the intrinsic potentialities of LGE/SVZ progenitors.

\section{Materials and Methods}

Animals. Experiments were performed on newborn [postnatal day 0 (P0) to P2] and young adult (1-2 months of age) CD1 strain mice (Charles River, Calco, Italy). For transplantation experiments, donor LGE/SVZ cells were dissected from transgenic mice overexpressing the enhanced green fluorescent protein (EGFP) under the control of the $\beta$-actin promoter (a generous gift from Dr. M. Okabe, Osaka University, Osaka, Japan) (Okabe et al., 1997). All experimental procedures were in accordance with the European Communities Council Directive of November 24, 1986 (86/609/EEC), the National Institutes of Health guidelines (no. 85-23.1985), the Italian law for care and use of experimental animals (DL116/92), and approved by the Italian Ministry of Health and the Bioethical Committee of the University of Turin and the Institutional Animal Care and Utilization Committee, University of Maryland.

Stereotaxic surgery and tracer injection. Stereotaxic injections were performed on newborn (P0-P2) and adult (2 months of age) mice as described previously (De Marchis et al., 2001). Briefly, newborn mice were anesthetized by hypothermia (total duration, 15-20 min), and their heads were immobilized on a custom neonatal stereotaxic apparatus maintained at $4^{\circ} \mathrm{C}$ during surgery. The skull was exposed by a skin incision, and small holes were drilled through. A total of $100 \mathrm{nl}$ of Fluorogold (FG) (Invitrogen, Eugene, OR) were injected at stereotaxic coordinates of $0 \mathrm{~mm}$ bregma, $0.8 \mathrm{~mm}$ lateral to sagittal sinus, and $1.5 \mathrm{~mm}$ depth, by means of a glass micropipette and a pneumatic pressure injection apparatus (Picospritzer II; General Valve, Fairfield, IL). After the micropipette was removed, the skin was sutured with $0.8 \mathrm{~mm}$ silk thread. The pups were quickly revitalized under a heat lamp and subsequently returned to the dam. Adult animals were anesthetized by intraperitoneal injection of sodium pentobarbital (Nembutal; $4-5 \mathrm{mg} / 100 \mathrm{mg}$ body weight in $0.9 \%$ saline solution), positioned in a stereotaxic apparatus (Stoelting, Wood Dale, IL), and maintained on a warm platform at $35^{\circ} \mathrm{C}$ to keep body temperature constant during anesthesia. The surgical procedure followed the same steps described for neonatal mice. Injections of $100 \mathrm{nl}$ of FG were made at stereotaxic coordinates of $0.5 \mathrm{~mm}$ anterior to bregma, 1 $\mathrm{mm}$ lateral to the sagittal sinus, and a depth of $2 \mathrm{~mm}$. After surgery, the animals were left under a heat lamp and constantly monitored until recovery.

Preparation of cell suspension and transplantation. Donor cells were dissected from embryo [embryonic day 15 (E15)], neonatal (P1-P2), and young adult (1 month of age) EGFP mice. The preparation of donor LGE/SVZ progenitor cells was performed as described previously (Carletti et al., 2002, 2004). Briefly, donor embryos were removed by caesar- ean section from deeply anesthetized timed-pregnant females at gestation day 15 (E15). The embryos were rapidly decapitated and dissected in PBS with $0.6 \%$ glucose (PBG) to isolate the forebrain. Under a dissecting microscope, the LGE was carefully dissected away from surrounding brain structures, and the collected tissue blocks were mechanically dissociated to a single-cell suspension in the same dissection medium without proteolytic agents. The obtained suspension was centrifuged and resuspended in the same solution at a final concentration of $3-5 \times 10^{4}$ cells/ $\mu$ l.

Neonatal mice were anesthetized by hypothermia, whereas adult mice were anesthetized with an intraperitoneal injection of ketamine (100 $\mathrm{mg} / \mathrm{kg}$; Ketavet; Gellini, Aprilia LT, Italy) and xylazine $(5 \mathrm{mg} / \mathrm{kg}$; Rompun; Bayer, Leverkusen, Germany). The anesthetized animals were decapitated and the forebrain was dissected in PBG and cut into $500 \mu \mathrm{m}$ coronal sections by means of a McIlwain tissue chopper. Under a highmagnification dissecting microscope, the SVZ of the lateral ventricle was carefully dissected away from surrounding brain structures, isolated, and mechanically dissociated to a single-cell suspension by means of a P200 Gilson pipette. In the case of P30 animals, before mechanical dissociation, SVZ fragments were incubated for $3 \mathrm{~min}$ in $0.025 \%$ trypsin and $0.265 \mathrm{~mm}$ EDTA. The cell suspension was then centrifuged $(1000 \times g$ for $7 \mathrm{~min}$ ) and resuspended in PBG at a final concentration of $3-5 \times 10^{4}$ cells/ $\mu$ l. Cell transplantation was performed on newborn and 2-monthold mice, following the same surgical procedure described previously for neuronal tracer injection. In all experiments, $2 \mu \mathrm{l}$ of the cell suspension were pressure-injected via a glass micropipette into both lateral ventricles of each recipient mouse.

Tissue processing and immunohistochemical procedures. At the end of the survival time ( $30 \mathrm{~d}$ after tracer injection, 30 or $60 \mathrm{~d}$ after cell transplantation), the mice were deeply anesthetized (as above) and transcardially perfused with $0.9 \%$ saline, followed by $4 \%$ paraformaldehyde in 0.1 $\mathrm{M}$ phosphate buffer (PB), $\mathrm{pH}$ 7.4. The brains were postfixed for $6 \mathrm{~h}$ in the same solution, cryoprotected in $30 \%$ sucrose in $0.1 \mathrm{M} \mathrm{PB}, \mathrm{pH} 7.4$, embedded in TissueTek OCT (optimal cutting compound) (Sakura Finetek, Torrance, CA), frozen on dry ice-cooled isopentane, and stored at $-80^{\circ} \mathrm{C}$ until sectioned. Free-floating series of coronal and/or parasagittal sections $(25 \mu \mathrm{m})$ were collected in multiwell dishes and stored at $-20^{\circ} \mathrm{C}$ in antifreeze solution until use.

Sections were incubated overnight at $4^{\circ} \mathrm{C}$ in primary antibody diluted in $0.01 \mathrm{M}$ PBS, $\mathrm{pH} 7.4,0.5 \%$ Triton $\mathrm{X}-100$, and $1 \%$ normal serum of the same species of the secondary antiserum, and then incubated for $1 \mathrm{~h}$ at room temperature in the appropriate secondary antibody. Sections were counterstained by incubation in $5 \mathrm{~nm}$ TOTO3 for $30 \mathrm{~min}$ at room temperature after immunohistochemical labeling and coverslipped with a DABCO [1,4-diazobicyclo-(2,2,2)octane]-based antifade mounting medium. The following primary antibodies were used: anti-PSA-NCAM (polysialylated form of the neural cell adhesion molecule) (1:2500; monoclonal mouse IgM; Ab-Cys, Paris, France), anti-DCX (doublecortin) (1:500; goat; C-18 antibody; Santa Cruz Biotechnology, Santa Cruz, CA), anti-GFAP (glial fibrillary acidic protein) (1:1000; monoclonal mouse IgG; Boehringer, Mannheim, Germany), anti-GFP (green fluorescent protein) (1:500; rabbit; Invitrogen), anti-calbindin D-28K (CB) (1:1000; mouse; Swant, Bellinzona, Switzerland), anti-calretinin (CR) (1:8000; rabbit; Swant), anti-tyrosine hydroxylase (TH) (1:1500; rabbit; Institut Jacques Boy, Reims, France), anti-neurocalcin (NC) (gift from Dr. H. Hidaka, Western Therapeutic Institute, Nagoya, Japan). Secondary antibodies used were as follows: anti-mouse and anti-rabbit Cy3 conjugated (1:800; Jackson ImmunoResearch, West Grove, PA); antigoat biotinylated (1:250; Vector, Burlingame, CA) followed by avidin Cy3 or Cy2 (1:800; Jackson ImmunoResearch). FG can be visualized using a wide-band ultraviolet excitation filter.

Photography and data analysis. All images were captured on a FluoView 500 confocal microscope (Olympus Instruments, San Francisco, $\mathrm{CA}$ ) fitted with standard excitation and emission filters for the visualization of FG, EGFP, Cy3, and TOTO3. Confocal image $z$-stacks were captured through the thickness of the slice at $1 \mu \mathrm{m}$ optical steps. The digital images were brightness, color, and contrast balanced, assembled into montages using CorelDraw 11 (Corel, Ottawa, Ontario, Canada) and LSM Image Browser (Zeiss, Oberkochen, Germany). 
A

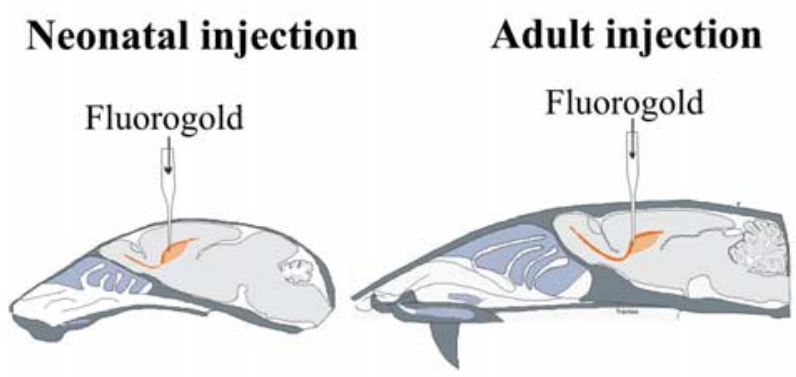

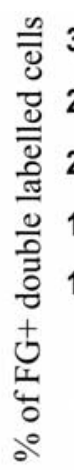

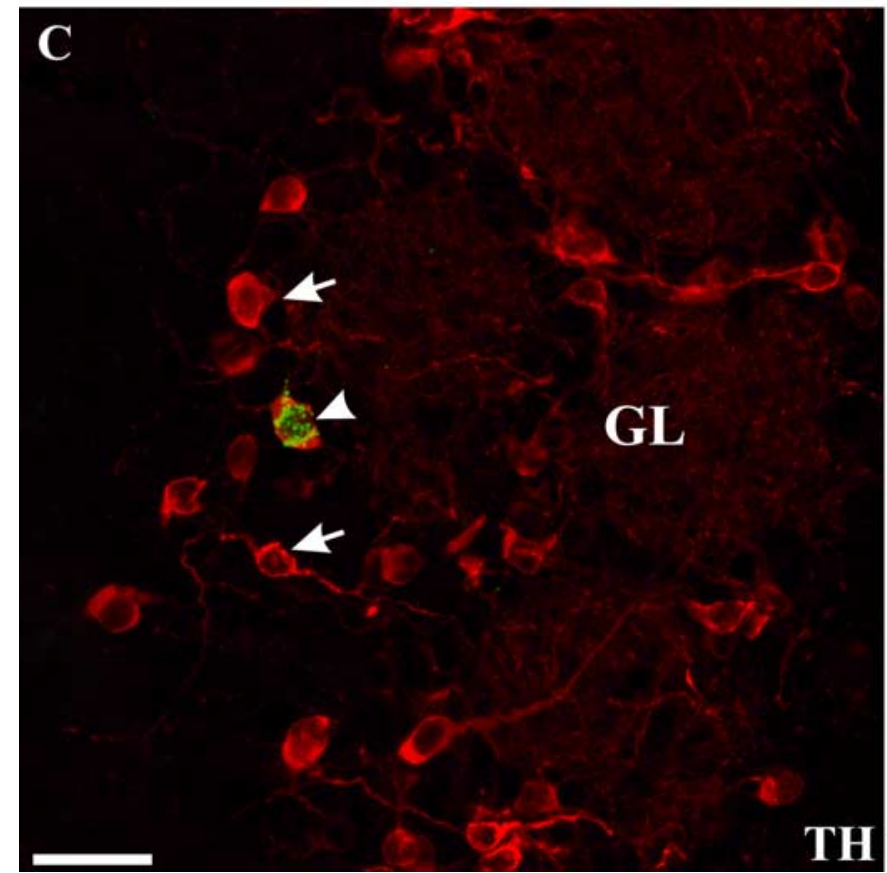

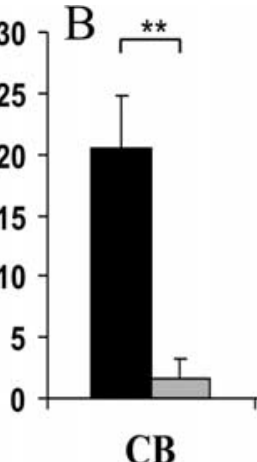
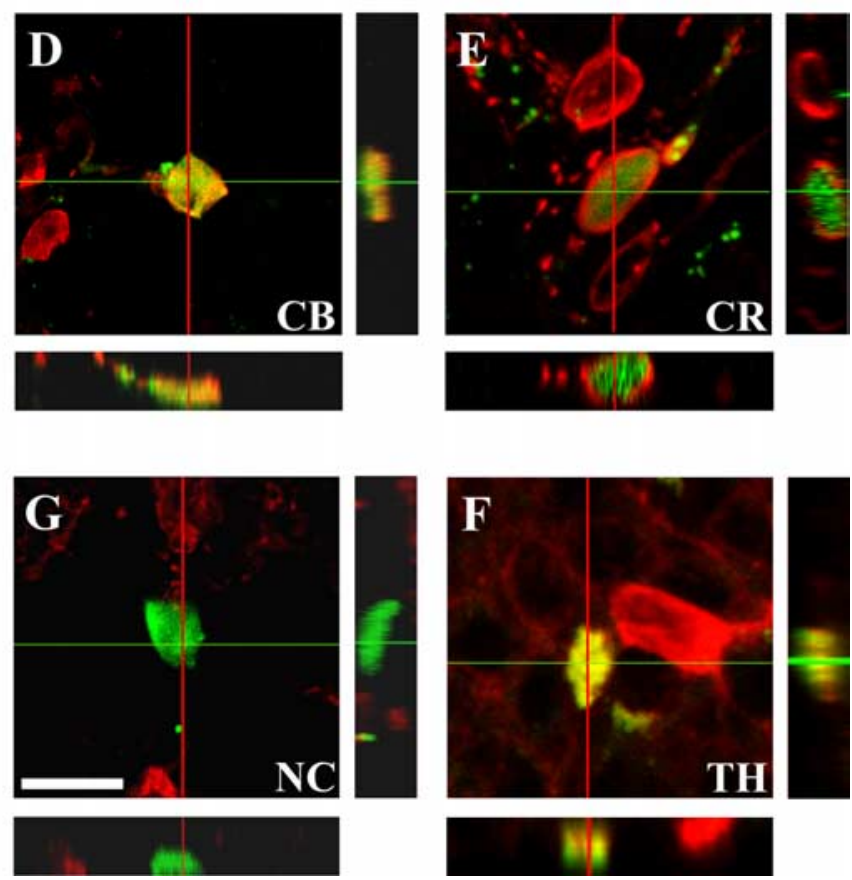

Figure 1. Neurochemical diversity of SVZ-derived cells (FG positive; green) generated at different ages. Double labeling shows as yellow in the overlay. $A$, Schematic drawings illustrating the FG injection site in newborn and adult mice. $B$, Quantification of FG-positive $\mathrm{GL}$ cells double-labeled for $\mathrm{CB}, \mathrm{CR}$, NC, or TH, among the total number of FG-positive cells counted in the GL at $30 \mathrm{~d}$ survival in newborn and adult injected animals. Error bars indicate SEM. ${ }^{*} p<0.05 ;{ }^{* *} p<0.01$. C, Olfactory bulb GL stained in red for TH; the arrows indicate single-labeled cells, whereas the arrowhead shows a FG-positive neuron expressing TH. D-G, Confocal analysis of FG-positive cells in sections labeled for CB, CR, NC, and TH. Scale bars: C, $30 \mu \mathrm{m}$; (in G) $D-G, 10 \mu \mathrm{m}$.

In both tracing and transplantation experiments, sections were examined systematically through the bulb and FG-positive or EGFPexpressing cells in the GL were assessed for double labeling. Cell quantifications were performed by counting all FG- or EGFP-labeled cells integrated in the OB GCL and GL on serial sections (1 serial section every 12; at least three animals for each experimental group). For each animal, the percentages of cells integrated into the GL and GCL, respectively, were calculated over the total number of cells counted.

To establish the neurochemical phenotype of labeled cells in both tracing and transplantation experiments, the percentage of FG- or EGFPlabeled cells integrated in the GL expressing either calbindin, calretinin, or TH was evaluated on confocal $z$-stacks captured through the thickness of the slice. Morphometric analysis (soma diameter and number of primary dendrites) was performed on confocal images using Image-Pro Plus software (Media Cybernetics, Silver Spring, MD). Statistical comparisons were conducted by Student's $t$ test or by multivariate ANOVA (MANOVA), followed by Bonferroni's post hoc comparison. Significance was established at $p \leq 0.05$. Data are presented as means \pm SEM. Statistics for the tracing and transplantation experiments were all performed on the mean values obtained for each animal, with $n$ being the number of animals.

\section{Results}

Selective neurochemical classes of periglomerular interneurons are generated during neonatal and adult $\mathrm{OB}$ neurogenesis

Interneurons in the OB GL are heterogeneous in their morphology, neurochemical phenotype, and synaptic properties (Shipley et al., 2004; Kosaka and Kosaka, 2005; Parrish-Aungst et al., 2007), whereas interneurons in the GCL, mostly represented by GABAergic granule cells, are more homogeneous. Little is known about the functional significance of the diversity in neurotransmitter/peptide for olfactory signal processing, and it is unclear whether the different chemically coded subclasses of cells are generated in specific time windows during development or in the adult. To elucidate the relative contribution of neonatal and adult OB neurogenesis to the different neurochemical subclasses of PG interneurons, we stereotaxically injected FG into the SVZ of newborn and adult mice (Fig. 1A) (De Marchis et al., 2001). One month after injection, the fraction of FG-labeled cells integrated in the GL or in the GCL was similar in both newborn $(7.8 \pm 2.2 \%$ of cells integrate in the GL; $n=6)$ and adult mice ( $9.7 \pm 1.1 \%$ of 
cells integrate in the GL; $n=4$ ). To examine the neurochemical phenotype of PG interneurons, we focused on the expression of three calcium-binding proteins (CB, CR, and NC) and TH (Fig. $1 C-G)$. Thirty days after FG injection, $20.6 \pm 4.3 \%$ of the FGpositive cells in the GL of newborn animals ( $n=5,236$ cells) were double labeled for calbindin, whereas in adult mice they were only $1.6 \pm 1.6 \%(n=4,154$ cells; $t$ test, $p<0.01)$ (Fig. $1 B)$. FG-stained/CR-immunoreactive cells were $8.9 \pm 3.4 \%(n=5$, 139 cells $)$ and $17.9 \pm 2.8 \%(n=5,212$ cells; $t$ test, $p \leq 0.05)$ in neonatal and adult animals, respectively (Fig. $1 B$ ). In contrast, none ( $0 \%$ neonatal injection; $n=3,156$ cells) or very few ( $1.3 \pm$ $0.7 \%$ adult injection; $n=3,135$ cells) FG-positive cells were also labeled by NC antibodies (Fig. $1 B$ ). Concerning the dopaminergic phenotype, FG injection into neonatal animals yielded only $0.9 \pm 0.6 \%$ TH-positive PG neurons ( $n=4,206$ cells), whereas double-labeled cells were $9.7 \pm 1.8 \%$ in adult animals $(n=5,155$ cells; $t$ test, $p<0.01$ ) (Fig. $1 B$ ), consistent with previous studies on the rat suggesting that the majority of TH cells are born between birth and adult (McLean and Shipley, 1988; Winner et al., 2002). These observations show that different subclasses of PG cells are generated in an age-dependent manner: $\mathrm{CB}$-expressing cells are preferentially produced during early life, whereas addition of newly generated CR- and TH-positive neurons is moderate in newborn animals, but proceeds at a higher pace during adult life (Fig. $1 B$ ). Our data suggest that SVZ progenitors of different developmental stages differentially contribute to specific subclasses of OB interneurons. SVZ progenitors may be autonomously committed to specific phenotypes or driven to undergo selective differentiation through interaction with extrinsic cues.

\section{The majority of transplanted EGFP cells become periglomerular and granule cells}

To investigate the age-related potential of SVZ progenitors and the relative contribution of intrinsic properties and environmental cues in the regulation of $\mathrm{OB}$ interneuron specification, we adopted a cell transplantation paradigm. We compared the fate of EGFP-tagged donor cells (Okabe et al., 1997) from neonatal and adult SVZ grafted to the SVZ of newborn or adult hosts (Fig. 2 A). In addition, transplantation of E15 LGE cells into newborn or adult hosts was performed to examine the behavior of embryonic precursors (Fig. 2A).

Donor cells were frequently observed along the injection track as well as within several adjacent areas of the forebrain. These cells displayed morphological features of both neuronal and glial lineages. The phenotypic repertoires produced by transplanted cells in extra-OB regions of the recipient brain were generally consistent with the donor age (i.e., more neurons were generated by LGE cells) and the engraftment site (i.e., oligodendrocytes were present along white matter tracts). However, given the specific goals of our experiments, the subsequent description will be focused on the cells that integrated into the SVZ-OB, which exclusively adopted neuronal identities.

Progenitors isolated from donors of different ages efficiently incorporated into the SVZ adjacent to lateral ventricle of both neonatal and adult recipients. Clusters of such cells could be found in this position up to 2 months after grafting, and EGFPpositive neuroblasts were seen migrating along the RMS. Numerous donor cells eventually integrated both into the accessory (data not shown) and main OB. The numbers of EGFP-positive cells counted in the $\mathrm{OB} 1$ or 2 months after transplantation were not statistically different $(p>0.05)$. Therefore results obtained at the two survival times were pooled for the subsequent analyses.
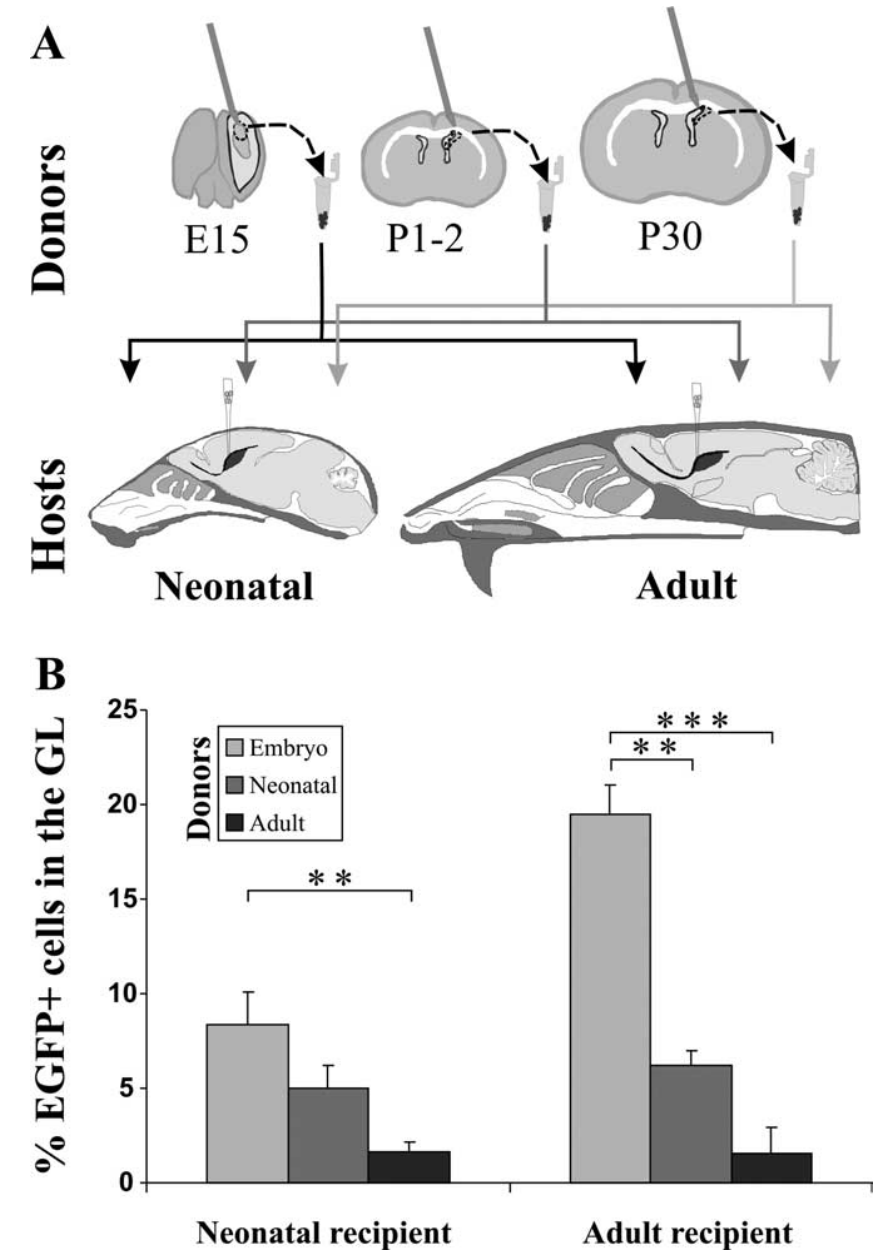

Figure 2. Homochronic and heterochronic transplantation to the neonatal and adult SVZ.A, Schematic drawings illustrating the transplantation experiments: donors cells derived from the LGE/SVZ of embryonic, neonatal, and adult mice were transplanted into the SVZ of neonatal and adult recipients. Analysis of the engrafted cells integrated into the $\mathrm{OB}$ was performed at 1 and 2 months of survival. $\boldsymbol{B}$, Integration rate of EGFP-positive cells into the $\mathrm{GL}$ in the different donorrecipient combinations. MANOVA analysis shows a significant effect of donor and host with interaction between them and no effect of survival time. Host effect was observed only in the case of embryo donors $(p<0.05$ ). Donor effect was observed both in adult (embryo vs newborn, $p>0.01$; embryo vs adult, $p>0.001$ ) and newborn (embryo vs adult, $p>0.01$ ) recipients. Error bars indicate SEM. ${ }^{* *} p<0.01 ;{ }^{* * *} p<0.001$.

EGFP-positive cells showed the typical morphological features and anatomical localization of fully differentiated $\mathrm{OB}$ interneurons (Fig. 3A-F) (Petreanu and Alvarez-Buylla, 2002). In neonatal hosts, the number of donor EGFP-positive cells integrated into the $\mathrm{OB}$ was similar with all donor ages from embryo to adult (embryo: $516 \pm 86$ cells, $n=11$; neonatal: $407 \pm 82$ cells, $n=9$; adult: $463 \pm 103$ cells, $n=9)$. In contrast, in adult recipients neonatal cells showed higher integration efficiency (1445 \pm 277 cells; $n=9$ ) compared with both embryo (613 \pm 138 cells; $n=11$ ) and adult donors ( $193 \pm 87$ cells; $n=5$; Bonferroni's post hoc test, $p<0.05$, neonatal vs embryo; $p<0.01$, neonatal vs adult). Moreover, transplants of neonatal SVZ neuroblasts were more effective in adult than in newborn recipients (Bonferroni's post hoc test, $p<0.01$ ). Because of higher proliferation rates or better survival capabilities, neonatal cells could have some competitive advantage versus their adult counterparts and be more efficiently incorporated in the SVZ of mature animals. However, the apparent decline of adult donor cell integration into adult 

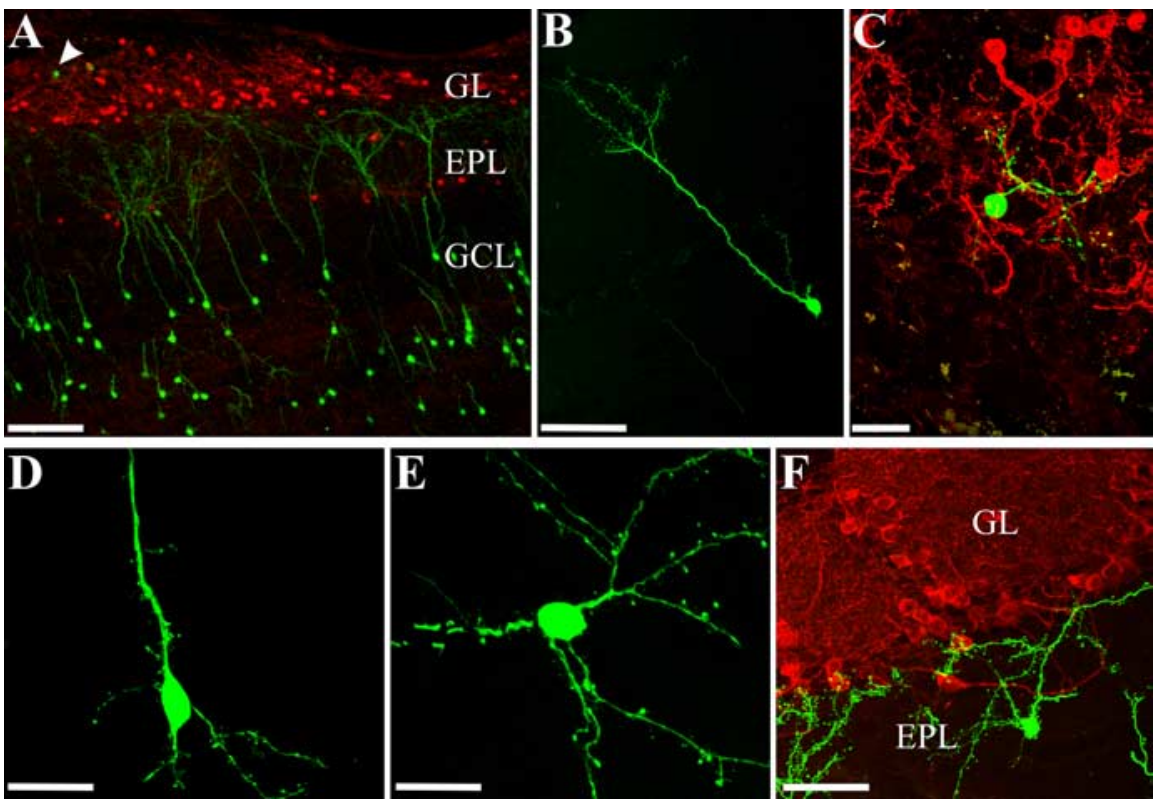

Figure 3. Morphological characterization of EGFP-positive cells in the OB. A, EGFP-positive cells (green) derived from neonatal donors integrated in the $\mathrm{OB}$ of an adult mouse 1 month after transplant in a tissue section double stained for CB (red). EGFPpositive cells are predominantly localized in the GCL. The arrowhead shows an EGFP-positive cell integrated in the GL. $\boldsymbol{B}, \boldsymbol{D}$, EGFP-positive GCs derived from LGE embryonic progenitors, integrated in the OB GCL. $E$, EGFP-positive nongranule cells derived from LGE embryonic progenitors, integrated in the OB GCL. $\boldsymbol{F}$, EGFP-positive cells derived from neonatal SVZ progenitors, integrated in the OBEPL, in a section double stained for TH. Scale bars: $\boldsymbol{A}, 100 \mu \mathrm{m} ; \boldsymbol{B}, \boldsymbol{F}, 50 \mu \mathrm{m} ; \boldsymbol{C}-\boldsymbol{E}, 20 \mu \mathrm{m}$.

hosts suggests that the juvenile milieu may provide more favorable conditions for the engraftment of these cells.

EGFP-positive cells were mainly localized in the GCL and less abundantly in the GL (Fig. $3 A$ ). In the GCL, the vast majority of donor cells were morphologically identifiable as mature GCs, characterized by a round or elongated cell body, a thick, long apical process that extended through the mitral cell layer (MCL) to form spine-rich apical dendrites in the external plexiform layer (EPL), and one or a few basal processes extending into the GCL (Petreanu and Alvarez-Buylla, 2002) (Fig. 3 B,D). Transplanted GCs were also found mixed with mitral cells in the MCL (data not shown). In addition to typical GCs, we also encountered EGFPpositive elements with larger polygonal cell bodies (soma diameter: $11.5 \pm 0.5 \mu \mathrm{m} ; n=8$; vs $9.1 \pm 0.4 \mu \mathrm{m} ; n=9 \mathrm{GCs} ; t$ test, $p<$ 0.01 ) and several processes (number of primary dendrites, $4.6 \pm$ $0.7 ; n=7$; vs $2.4 \pm 0.2 ; n=9 \mathrm{GCs} ; t$ test, $p<0.05$ ) radiating in the GCL (Fig. 3E). Based on their morphology, these cells could be ascribed to nongranule cells types such as Blanes cells and/or Golgi cells, which are present in low density in the GCL of the adult OB (for review, see Shipley et al., 2004). These neurons, found in the large majority of animals transplanted with embryonic cells independently from the host age, were only occasionally observed with neonatal and adult donors.

Donor cells integrated in the GL showed the typical morphology of PG neurons (Fig. 3C). A few morphologically differentiated EGFP-positive elements were also found immediately deep to the GL, in the EPL. The round-shaped bodies of these cells were either localized on the EPL/GL boundary, with horizontally oriented spine-rich dendrites budding from the soma in opposite directions, or in the superficial EPL, with multiple spiny processes reaching the glomerular shell (Fig. $3 F$ ). These rare EPL cells were preferentially observed in animals transplanted with either embryonic or neonatal progenitors, regardless of the recipient age. On the whole, our findings suggest that, along with the main populations of GC and PG cells, LGE/SVZ precursors of different ages may also contribute to other classes of $\mathrm{OB}$ interneurons.

\section{Age-related changes in the integration rate of transplanted cells into the GCL versus the GL}

The ratio of EGFP cells integrating into the GCL versus GL varied between the host and donor combinations with the majority of cells integrating into the former, as expected (Bayer, 1983; Luskin, 1993; Lois and Alvarez-Buylla, 1994). Adult-derived SVZ donors rarely yielded cells that integrated in the GL of newborn or adult host $(1.6 \pm 0.6 \%$ in newborn, $n=9 ; 1.5 \pm$ $1.4 \%$ in adult, $n=5)$, whereas embryonic $(8.4 \pm 1.7 \%$ in newborn, $n=11 ; 19.5 \pm$ $1.5 \%$ in adult hosts, $n=9$ ) or neonatal ( $5 \pm 1.2 \%$ in newborn, $n=9 ; 6.2 \pm 0.8 \%$ in adult hosts, $n=11$ ) donor transplants generated larger fractions of GL neurons (Fig. $2 B$ ). In both newborn and adult recipients, there was a significant effect of donor age on transplant (Bonferroni's post hoc test, for newborn recipients, $p<0.01$, embryo vs adult; for adult recipients, $p<$ 0.01 , embryo vs neonatal, and $p<0.001$, embryo vs adult donors). Embryonic LGE cells, but not neonatal or adult SVZ cells, were more efficient in integrating into the GL of adult compared with newborn hosts $(p<0.05)$ (Fig. $2 B$ ). Together, these observations show a progressive decrease in the number of cells targeted to the GL depending on the donor age of the transplanted progenitors, from embryo to adult. However, neonatal and adult cells consistently behave according to their donor origin, whereas embryonic cells are partially influenced by the host environment.

\section{Transplanted embryonic and neonatal progenitors exhibit different PG cell lineage potential}

Our previous studies suggested that at least some of the SVZ progenitors are committed to a GAD65-positive phenotype as early as the SVZ, and there does not appear to be any agedependent differences for selecting the GAD lineage (De Marchis et al., 2004). To investigate whether cells in the SVZ are committed to differentiate into other neurochemical phenotypes at different ages, we analyzed the expression of PG cell markers in transplanted cells. Because of the very low number of EGFPpositive cells integrated in the GL of animals that received adult SVZ donors, these specimens were not included in the quantitative analysis. The percentage of EGFP-labeled cells expressing either $\mathrm{CB}, \mathrm{CR}$, or TH was evaluated in the GL of newborn and adult mice transplanted with embryonic or neonatal donors (Fig. 4). Although the number of donor PG cells varied considerably from case to case, the fractions of such cells belonging to different phenotypes at 1 or 2 months after transplantation did not reveal any clear trend and were not statistically different $(p>0.05)$. Therefore, although we cannot completely rule out the possibility that the frequencies of certain phenotypes could change slightly over time, we decided to pool data from the two survival times also for this analysis.

Embryonic donors yielded numerous EGFP cells expressing 
CB $(25.9 \pm 4.9 \%$ in newborn, $n=5,349$ cells; $16.5 \pm 3.9 \%$ in adult, $n=8,216$ cells) (Fig. 4A). Neonatal cells, transplanted to either newborn or adult hosts, rarely acquired this phenotype (namely, of $5.1 \pm$ $3.1 \%$ in newborn, $n=5,52$ cells; $2.8 \pm$ $1.4 \%$ in adult recipients, $n=10,349$ cells) (Fig. 4A). Therefore, transplanted embryonic progenitors show a higher potentiality than neonatal donors to develop CBpositive PG neurons $(p<0.01)$ and this phenomenon is independent of host age $(p>0.05)$. Virtually no embryonic LGE cells developed CR-positive PG neurons ( $0 \%$ in newborn, $n=5,207$ cells; $1.6 \pm$ $1.6 \%$ in adult recipients, $n=9,299$ cells), whereas neonatal progenitors gave rise to CR cells both in newborn $(12.8 \pm 10.2 \%$, $n=4,58$ cells $)$ and adult $(8.7 \pm 1.6 \%, n=$ 10,345 cells) recipients ( $p<0.01$ embryo vs neonatal donors in adult recipient) (Fig. $4 B$ ). Finally, the analysis of TH expression in EGFP-positive cells derived from either embryonic ( $5.4 \pm 3.6 \%$ in newborn, $n=5$, 214 cells; $2.2 \pm 1.5 \%$ in adult hosts, $n=9$, 293 cells) or newborn $(7.3 \pm 4.7 \%$ in newborn, $n=4,35$ cells; $7 \pm 3.1 \%$ in adult hosts, $n=10$, 332 cells) mice did not show any significant differences between donors and/or recipients (Fig. 4C). Hence, differentiation of donor cells into CR- or CBpositive $\mathrm{PG}$ interneurons depends on the age of the donor cells, and it is minimally influenced by the host. The generation of TH-positive PG cells also showed a similar trend, being more frequent with neonatal SVZ than embryonic LGE progenitors.

\section{Discussion}

Local circuit interactions within the $\mathrm{OB}$ modulate the activity of major output neurons. This function is produced by a variety of interneurons and is essential for olfactory processing. Recent reports have shown that $\mathrm{OB}$ interneurons are generated and functionally integrated into local circuits throughout life (Doetsch and Hen, 2005). In addition, some of the molecular mechanisms underlying the production and diversity of $\mathrm{OB}$ interneurons have been unraveled (Hack et al., 2005; Kohwi et al., 2005; Waclaw et al., 2006). These studies indicate that different $\mathrm{OB}$ interneuron phenotypes derive from distinct sets of progenitor cells, which become lineage restricted in the SVZ or in the RMS. Our in vivo cell labeling in neonate and adult, together with embryonic and neonatal transplants, strongly suggests that the potentiality of SVZ progenitors is also modulated in time, so that specific neurochemical classes of PG interneurons are preferentially generated at different ages.

Although the adult SVZ-OB system retains many immature features, substantial molecular and functional changes character-
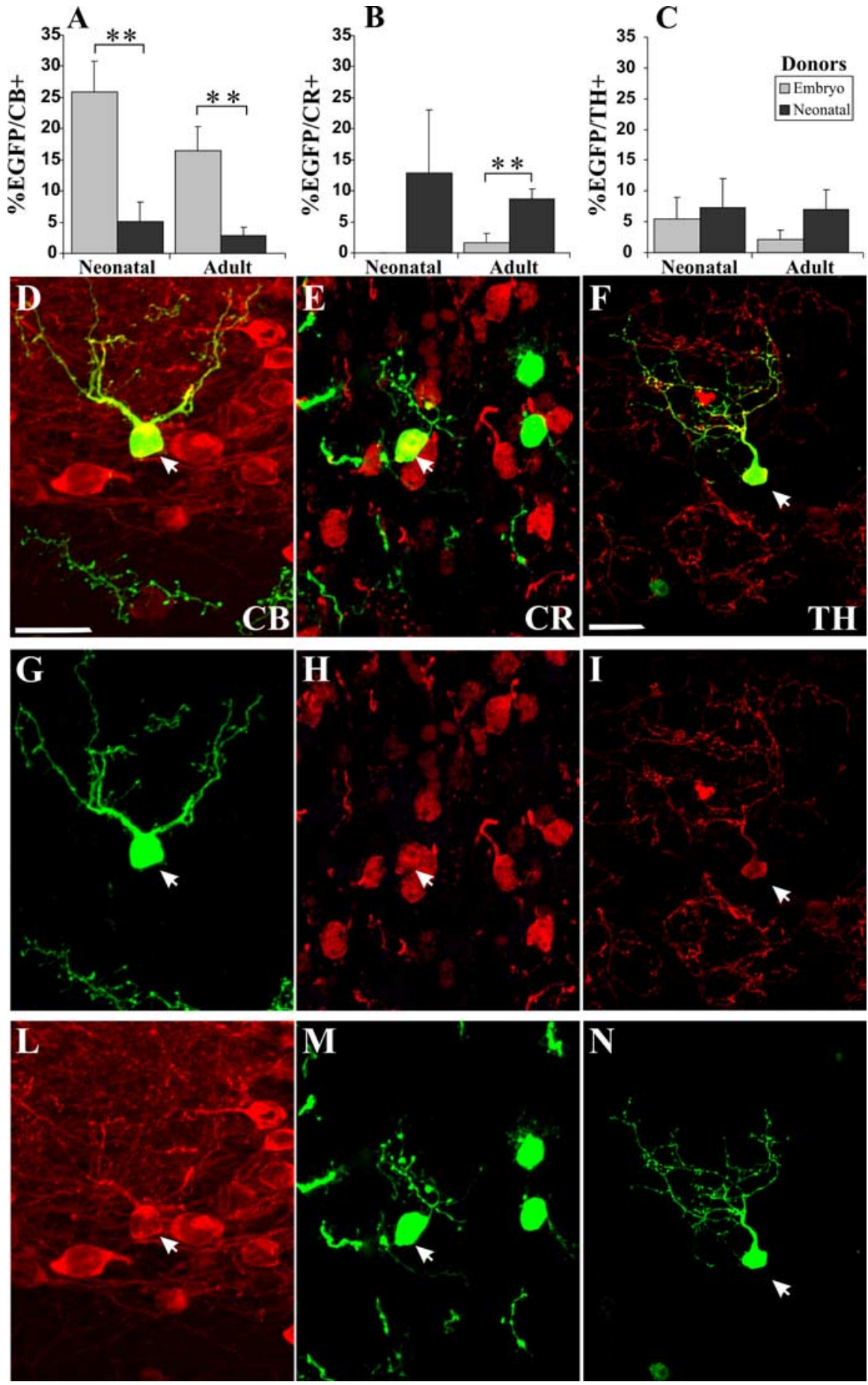

Figure 4. Neurochemical phenotype of EGFP-positive cells integrated in the $O B G L$ in the different donor-recipient combinations. $\boldsymbol{A}-\boldsymbol{C}$, Quantification of percentage of EGFP-positive cells integrated in the GL double stained for $C B(\boldsymbol{A}), \mathrm{CR}(\boldsymbol{B})$, and TH $(\boldsymbol{C})$. Comparison of the results obtained transplanting embryonic and neonatal progenitors into newborn and adult mice. $\boldsymbol{D}-\boldsymbol{N}$, Representative images of EGFP-positive cells double stained for $C B(\boldsymbol{D}, \boldsymbol{G}, \boldsymbol{L}), C R(\boldsymbol{E}, \boldsymbol{H}, \boldsymbol{M})$, and $\mathrm{TH}(\boldsymbol{F}, \boldsymbol{I}, \boldsymbol{N})$. Error bars indicate $\operatorname{SEM}$. ${ }^{* *} p<0.01$. Scale bars: (in D) $\boldsymbol{D}, \boldsymbol{E}, \boldsymbol{G}, \boldsymbol{H}, \boldsymbol{L}, \boldsymbol{M}, 20 \mu \mathrm{m}$; (in $\left.\boldsymbol{F}\right) \boldsymbol{F}, \boldsymbol{I}, \boldsymbol{N}, 20 \mu \mathrm{m}$.

ize its development (Malun and Brunjes, 1996; Bailey et al., 1999; Peretto et al., 2005). Previous reports indicated that SVZ cells from adult mice transplanted into the adult SVZ migrate into the host $\mathrm{OB}$ and differentiate into GC and PG interneurons (Lois and Alvarez-Buylla, 1994). Progenitors from the LGE grafted to the hosts of different ages also show the same migratory behavior and developmental potential (Wichterle et al., 1999, 2001). Accordingly, we found that donors of all ages transplanted to the SVZ of 
both newborn and adult mice were able to migrate along the RMS and to acquire $\mathrm{OB}$ interneuron phenotypes, although with different efficiency in term of total numbers of integrated cells. As expected, based on the normal behavior of naive progenitors (Bayer, 1983; Luskin 1993; Lois and Alvarez-Buylla, 1994), higher amounts of transplanted cells engrafted in the GCL than in the GL. However, quantitative analysis of the integration rate of transplanted cells into each layer showed that progenitors of diverse ages exhibit different behaviors, with a progressive decrease in the number of cells targeted to the GL from embryo to adult. This effect was mostly independent from the age of the host, although the fraction of embryonic donors that engrafted in the GL was bigger in adult than neonatal recipients. These results indicate that the integration of embryonic, but not newborn or adult, progenitors is conditioned by maturational changes in the host environment, likely reflecting a less committed state of LGE progenitors.

The fraction of PG cells observed in tracing and transplantation experiments was somewhat different. Indeed, variable estimations within the same range as our data have been reported by others in different experimental conditions (Luskin, 1993; Belluzzi et al., 2003; Hack et al., 2005). In particular, viral transfection approaches revealed that the amount of labeled PG cells is higher after injections in the RMS than in the SVZ (Hack et al., 2005). In line with these findings, the higher frequency of PG cells labeled in our tracing experiments might be attributed to diffusion or spillover of the tracer from the SVZ injection site along the adjacent caudal portion of the RMS.

In addition to GC and PG cells, EGFP-positive elements integrated in the host OB displayed morphological features reminiscent of other types of interneurons in the GCL (i.e., "Blanes cells") (Pressler and Strowbridge, 2006) and EPL. Although quantitative analyses were not performed on these cells, they were clearly more frequent in animals transplanted with juvenile progenitors, suggesting that along with the main populations of GC and PG cells, precursors from the LGE and SVZ may also contribute to other classes of $\mathrm{OB}$ interneurons. The generation of these interneuron types seems to be restricted to embryonic or postnatal ontogenesis. However, it is noteworthy that the adult OB still provides permissive conditions for their proper differentiation and integration.

Another significant age-related difference concerned the production of neurochemically distinct types of interneurons. Our results not only reveal differences in the neurogenic process occurring in the $\mathrm{OB}$ at distinct ages but also support the recent view that SVZ progenitors are intrinsically directed toward specific lineages (Hack et al., 2005; Kohwi et al., 2005; Waclaw et al., 2006).

The analysis of the neurochemical phenotypes of PG neurons after dye injection or transplantation discloses some consistent trends in the generation of specific GL interneuron types at different ontogenetic stages. CB-expressing neurons are mainly produced in juvenile individuals, whereas CR-positive cells are more frequently generated in adults. TH-positive cells are generated at low levels in the embryo/neonate and at higher rates in the adult, in agreement with previous studies showing that the greatest numbers of TH cells occur in the postnatal/adult OB (McLean and Shipley, 1988; Winner et al., 2002). The results of in situ labeling may be attributed either to age-dependent changes in the inherent features of SVZ progenitors, or to progressive modifications in the $\mathrm{OB}$ milieu that influence the final maturation of newly born neurons. Indeed, although different types of neocortical inhibitory interneurons derive from distinct progenitor pools (Wonders and Anderson, 2006), those of the cerebellum originate from multipotent precursors that acquire their mature identities under the influence of local instructive cues (Leto et al., 2006). To discriminate between these possibilities, we transplanted LGE/SVZ progenitors into homochronic or heterochronic environments. Grafted cells preferentially acquired phenotypes typical of their origin, thus showing that their mature identities are primarily determined by their intrinsic properties.

These results indicate that adult neurogenesis does not reflect a simple turnover of the whole interneuron population of the GL, but it may serve to finely modulate the relative amounts of distinct $\mathrm{OB}$ neuron subpopulations. This is strengthened by comparison of the results obtained by FG injection into adult mice with data on the percentages of the different subpopulations in the OB GL (Parrish-Aungst et al., 2007). Thus, considering the "steady-state" cell numbers for each of the neurochemical subpopulations with the proportion of newly generated cells yields a ratio (proportion of newly generated cells expressing each phenotype/proportion of cells in the GL expressing that phenotype) of 1.0 for $\mathrm{TH}$ and 0.6 for $\mathrm{CR}$, suggesting a higher rate of cell renewal in adult. In contrast, values for $\mathrm{CB}$ and $\mathrm{NC}$ are 0.2 and 0.1 , respectively, suggesting a low rate of cell renewal for these populations in adult. Our previous estimate of GAD65-positive cells generated by the SVZ (De Marchis et al., 2004) and the total adult GL population of GAD65-positive neurons yield a ratio of 1.25. This suggests a TH-positive cell in adult is 10 times, and a GAD65-positive cell 12 times, more likely to be renewed from SVZ progenitors than an NC-positive cell.

Together, our findings support the conclusion that the population of SVZ progenitors with a PG fate is composed of a heterogeneous pool of neurochemically specified elements, and indicate that the relative contribution of these different subsets changes with time. However, results obtained after SVZ progenitor transplantation partially diverge from those obtained by dye labeling. In particular, different percentages of CB- or $\mathrm{TH}$ positive cells are found when dye injections in the neonate are compared with transplantation of neonatal SVZ. These quantitative differences may be well related to the two experimental approaches. Indeed, the procedures of dissociation and transplantation may favor the survival and/or engraftment of certain cell types, whereas dye injection and uptake may be differentially efficient on distinct progenitor subsets. Such "selective" mechanisms would support the idea of intrinsically different subsets of progenitor cells destined to generate the variety of PG cell phenotypes. In any case, although the absolute numbers observed in the transplants cannot be compared with those obtained by tracing endogenous precursors, both sets of results are consistent in showing some clear trends in the age-dependent production of specific phenotypes. SVZ progenitors preferentially adopt the CB phenotype during embryonic life, whereas acquisition of the same identity gradually becomes less frequent during postnatal development and adulthood. In contrast, the production of CRand TH-expressing PG cells follows an opposite trend with a progressive increase with age. Therefore, despite their inherent methodological differences, both experimental approaches support the conclusion that different categories of PG cells are generated according to an age-specific schedule.

The functional impact to the OB circuit of "stable" (e.g., NC and $\mathrm{CB}$ ) and "transient" (e.g., CR, TH, and GAD) neuron populations in the adult GL is unclear. Given the specific role of calcium-binding proteins in regulating neuronal signaling and activity by buffering intracellular calcium (Berridge et al., 2003), it is tempting to speculate that differential renewal rates of CB- or 
CR-positive PG interneurons may be functional to some agespecific requirement in the processing of olfactory information. The observation that transplanted cells preferentially adopt neurochemical traits typical of their donor origin indicates that the age-specific repertoire of interneuron phenotypes is regulated by modulating the specific features of undifferentiated SVZ progenitors and not mature neurons. It remains to be elucidated whether these features reflect the execution of a genetically determined developmental schedule or they can be modified in response to individual experience.

\section{References}

Alvarez-Buylla A, Lim DA (2004) For the long run: maintaining germinal niches in the adult brain. Neuron 41:683-686.

Bailey MS, Puche AC, Shipley MT (1999) Development of the olfactory bulb: evidence for glia-neuron interactions in glomerular formation. J Comp Neurol 415:423-448.

Baker H, Liu N, Chun HS, Saino S, Berlin R, Volpe B, Son JH (2001) Phenotypic differentiation during migration of dopaminergic progenitor cells to the olfactory bulb. J Neurosci 21:8505-8513.

Bayer SA (1983) ${ }^{3} \mathrm{H}$-thymidine-radiographic studies of neurogenesis in the rat olfactory bulb. Exp Brain Res 50:329-340.

Belluzzi O, Benedusi M, Ackman J, LoTurco JJ (2003) Electrophysiological differentiation of new neurons in the olfactory bulb. J Neurosci 23:10411-10418.

Berridge MJ, Bootman MD, Roderick HL (2003) Calcium signalling: dynamics, homeostasis and remodelling. Nat Rev Mol Cell Biol 4:517-529.

Carleton A, Petreanu LT, Lansford R, Alvarez-Buylla A, Lledo PM (2003) Becoming a new neuron in the adult olfactory bulb. Nat Neurosci 6:507-518.

Carletti B, Grimaldi P, Magrassi L, Rossi F (2002) Specification of cerebellar progenitors after heterotopic-heterochronic transplantation to the embryonic CNS in vivo and in vitro. J Neurosci 22:7132-7146.

Carletti B, Grimaldi P, Magrassi L, Rossi F (2004) Engraftment and differentiation of neocortical progenitor cells transplanted to the embryonic brain in utero. J Neurocytol 33:309-319.

De Marchis S, Fasolo A, Shipley M, Puche A (2001) Unique neuronal tracers show migration and differentiation of SVZ progenitors in organotypic slices. J Neurobiol 49:326-338.

De Marchis S, Temoney S, Erdelyi F, Bovetti S, Bovolin P, Szabo G, Puche AC (2004) GABAergic phenotypic differentiation of a subpopulation of subventricular derived migrating progenitors. Eur J Neurosci 20:1307-1317.

Doetsch F, Hen R (2005) Young and excitable: the function of new neurons in the adult mammalian brain. Curr Opin Neurobiol 15:121-128.

Hack MA, Saghatelyan A, de Chevigny A, Pfeifer A, Ashery-Padan R, Lledo PM, Gotz M (2005) Neuronal fate determinants of adult olfactory bulb neurogenesis. Nat Neurosci 8:865-872.

Jankovski A, Sotelo C (1996) Subventricular zone-olfactory bulb migratory pathway in the adult mouse: cellular composition and specificity as determined by heterochronic and heterotopic transplantation. J Comp Neurol 371:376-396.

Kohwi M, Osumi N, Rubenstein JL, Alvarez-Buylla A (2005) Pax6 is required for making specific subpopulations of granule and periglomerular neurons in the olfactory bulb. J Neurosci 25:6997-7003.

Kosaka K, Toida K, Aika Y, Kosaka T (1998) How simple is the organization of the olfactory glomerulus? The heterogeneity of so-called periglomerular cells. Neurosci Res 30:101-110.
Kosaka T, Kosaka K (2005) Structural organization of the glomerulus in the main olfactory bulb. Chem Senses 30:107-108.

Lemasson M, Saghatelyan A, Olivo-Marin JC, Lledo PM (2005) Neonatal and adult neurogenesis provide two distinct populations of newborn neurons to the mouse olfactory bulb. J Neurosci 25:6816-6825.

Leto K, Carletti B, Williams IM, Magrassi L, Rossi F (2006) Different types of cerebellar GABAergic interneurons originate from a common pool of multipotent progenitor cells. J Neurosci 26:11682-11694.

Lois C, Alvarez-Buylla A (1994) Long-distance neuronal migration in the adult mammalian brain. Science 264:1145-1148.

Luskin MB (1993) Restricted proliferation and migration of postnatally generated neurons derived from the forebrain subventricular zone. Neuron 11:173-189.

Magavi SSP, Mitchell BD, Szentirmai O, Carter BS, Macklis JD (2005) Adult-born and preexisting olfactory granule neurons undergo distinct experience-dependent modifications of their olfactory responses in vivo. J Neurosci 25:10729-10739.

Malun D, Brunjes PC (1996) Development of olfactory glomeruli: temporal and spatial interactions between olfactory receptor axons and mitral cells in opossums and rats. J Comp Neurol 368:1-16.

McLean JH, Shipley MT (1988) Postmitotic, postmigrational expression of tyrosine hydroxylase in olfactory bulb dopaminergic neurons. J Neurosci 8:3658-3669.

Okabe M, Ikawa M, Kominami K, Nakanishi T, Nishimune Y (1997) "Green mice" as a source of ubiquitous green cells. FEBS Lett 407:313-319.

Parrish-Aungst S, Shipley MT, Erdelyi F, Szabo G, Puche AC (2007) Quantitative analysis of neuronal diversity in the mouse olfactory bulb. J Comp Neurol, in press.

Peretto P, Giachino C, Aimar P, Fasolo A, Bonfanti L (2005) Chain formation and glial tube assembly in the shift from neonatal to adult subventricular zone of the rodent forebrain. J Comp Neurol 487:407-427.

Petreanu L, Alvarez-Buylla A (2002) Maturation and death of adult-born olfactory bulb granule neurons: role of olfaction. J Neurosci 22:6106-6113.

Pressler RT, Strowbridge BW (2006) Blanes cells mediate persistent feedforward inhibition onto granule cells in the olfactory bulb. Neuron 49:889-904.

Shipley MT, Ennis M, Puche AC (2004) The olfactory system. In: The rat nervous system, Ed 3 (Paxinos G, ed), pp 921-962. New York: Elsevier.

Stenman J, Toresson H, Campbell K (2003) Identification of two distinct progenitor populations in the lateral ganglionic eminence: implications for striatal and olfactory bulb neurogenesis. J Neurosci 23:167-174.

Waclaw RR, Allen II ZJ, Bell SM, Erdelyi F, Szabo G, Potter SS, Campbell K (2006) The zinc finger transcription factor Sp8 regulates the generation and diversity of olfactory bulb interneurons. Neuron 49:503-516.

Wichterle H, Garcia-Verdugo JM, Herrera DG, Alvarez-Buylla A (1999) Young neurons from medial ganglionic eminence disperse in adult and embryonic brain. Nat Neurosci 2:461-466.

Wichterle H, Turnbull DH, Nery S, Fishell G, Alvarez-Buylla A (2001) In utero fate mapping reveals distinct migratory pathways and fates of neurons born in the mammalian basal forebrain. Development 128:3759-3771.

Winner B, Cooper-Kuhn CM, Aigner R, Winkler J, Kuhn HG (2002) Longterm survival and cell death of newly generated neurons in the adult rat olfactory bulb. Eur J Neurosci 16:1681-1689.

Wonders CP, Anderson SA (2006) The origin and specification of cortical interneurons. Nat Rev Neurosci 7:687-696. 